\title{
Collagen-targeting parathyroid hormone-related peptide promotes collagen binding and in vitro chondrogenesis in bone marrow-derived MSCs
}

\author{
XIAO-CHENG WU* , BO HUANG ${ }^{*}$, JIAN WANG, CHANG-QING LI and YUE ZHOU \\ Department of Orthopedics, Xinqiao Hospital, The Third Military Medical University, Chongqing, P.R. China
}

Received October 17, 2012; Accepted December 6, 2012

DOI: $10.3892 /$ ijmm.2012.1219

\begin{abstract}
Parathyroid hormone-related peptide (PTHrP) is an important inductive factor during chondrogenesis of mesenchymal stem cells (MSCs). PTHrP induces chondrogenesis and suppresses hypertrophy, yet the lack of an efficient delivery system limits its use for cartilage tissue engineering in clinical application. In this study, a peptide of 7 amino acids was first used to engineer PTHrP to construct a collagen-targeting system. This peptide functioned as a collagen-binding domain (CBD) to specially target the PTHrP to collagen. ELISA assay was used to determine the collagen-binding ability of CBD-PTHrP. The effect of CBD-PTHrP on chondrogenesis was measured by an in vitro pellet assay in bone marrow-derived MSCs (BM-MSCs). As expected, the CBD peptide promoted the binding of CBD-PTHrP to collagen when compared to NAT-PTHrP. Furthermore, the recombinant protein CBD-PTHrP induced the expression of COL2A1 and Sox-9, inhibited the expression of COL1A1 at the mRNA and protein levels as effectively as NAT-PTHrP. Safranin-O and immunohistochemistry for collagen types I, II, X and Sox-9 generally paralleled qRT-PCR and western blotting findings with minor variations. In conclusion, our study demonstrated that CBD-PTHrP is a collagen-targeting system and promotes in vitro chondrogenesis in BM-MSCs. We suggest that this is an efficient delivery system for cartilage tissue engineering in clinical application.
\end{abstract}

Correspondence to: Dr Yue Zhou, Department of Orthopedics, Xinqiao Hospital, The Third Military Medical University, Chongqing 400037, P.R. China

E-mail: happyzhou@vip.163.com

*Contributed equally

Key words: parathyroid hormone-related peptide, collagen-binding domain, collagen binding, chondrogenesis, bone marrow-derived mesenchymal stem cells

\section{Introduction}

Articular cartilage is a highly organized soft tissue. An articular cartilage defect is an area of damaged or missing cartilage (1). Although often caused by acute trauma, the defect may also occur as a result of osteoarthritis, osteonecrosis, osteochondritis dissecans and other pathologies (2). These degenerative joint diseases affect more than a third of the world population, and disorders of articular cartilage, in general, account for more than half of all chronic conditions in individuals aged 60 years and over (3). Therefore, optimized treatment strategies for articular cartilage lesions are of a high socio-economic importance. Symptomatic cartilage defects require surgical treatment such as microfracture, pridie drilling and abrasion arthroplasty, autologous chondrocyte transplantation, and the transfer of both autologous or allogeneic osteochondral transplants (4). Although the development of surgical techniques in cartilage has been extensively investigated $(5,6)$, there is currently no surgical method available for cartilage injuries that can prevent its early onset (7). Tissue engineering offers promising new approaches that have the potential to provide such therapies.

Mesenchymal stem cells (MSCs), which can be easily isolated in a non-invasive and abundant manner from various tissues such as the bone marrow, bone, adipose tissue, muscle, synovium, periosteum, perichondrium and many adult tissues (8), are capable of self-renewal and differentiation into a variety of cell lineages, including chondrocytes, osteoblasts and adipocytes $(9,10)$. MSCs have been identified in healthy and diseased cartilage, and appear to retain at least some potential to regenerate cartilage in vivo $(11,12)$. Due to these advantages, MSCs are attractive targets for manipulation in the goal of cartilage regeneration.

Chondrocyte proliferation and differentiation toward hypertrophy are the main challenges for cartilage regeneration from MSCs $(13,14)$. Various factors for chondrogenesis of MSCs have been developed, including parathyroid hormonerelated protein (PTHrP) (15). PTHrP, first identified as a factor involved in humoral hypocalcemia of malignancy (16), maintains the function of proliferating chondrocytes and inhibits chondrocyte differentiation toward hypertrophy in the growth plate through the PTHrP-Indian hedgehog (IHH) axis $(17,18)$. This anti-hypertrophic activity has been shown 
to result from binding of the N-terminus of PTHrP to its cell surface receptor (PTH1R), activating Sox9 (19). PTHrP also stimulates proliferation of endochondral chondrocytes and inhibits apoptosis, partly via induction of Bcl-2 (20). Therefore, PTHrP may be a therapeutic factor in the production of MSC-derived tissue-engineered cartilage for use in cartilage repair.

Collagen is the ubiquitous component of the tight network of glycoproteins, collagen IV and proteoglycans in basement membranes (21), and is widely dispersed in the articular site (22). Therefore, collagen could be a potential target for PTHrP which could be retained and enriched at the injured site, enhancing the efficacy of cartilage regeneration. A polypeptide TKKTLRT named collagen-binding domain (CBD) peptide was derived from von Willebrand factor (vWF). Epidermal growth factor, transforming growth factor-1 and basic fibroblast growth factor have been added along with a collagen-binding peptide and the results showed that CBD could specifically bind to native collagen and the modified growth factors achieved better repair ability compared to the native growth factors at the same concentration (23-25). Here, we constructed a collagen-based PTHrP-targeting system, and the effect on chondrogenesis was tested by in vitro pellet assay in bone marrow-derived (BM)-MSCs.

\section{Materials and methods}

Isolation and expansion of BM-MSCs. MSCs were isolated from fresh bone marrow samples obtained from patients undergoing total hip replacement or iliac bone graft harvest, as described elsewhere $(26,27)$. Briefly, cells were fractionated on a Ficoll-Paque Plus density-gradient (GE Healthcare), and the low-density cell fraction was washed and seeded in expansion medium consisting of high-glucose Dulbecco's modified Eagle's medium/F12 (DMEM/F12) and 10\% FBS (Gibco-BRL, Carlsbad, CA, USA). Nonadherent material was removed after 24-48 h. For expansion, cells were replated at a density of $5 \times 10^{3}$ cells $/ \mathrm{cm}^{2}$ and used at passage 3 .

Engineering and preparation of NAT-PTHrP and CBD-PTHrP. Engineering and preparation of NAT-PTHrP and CBD-PTHrP were performed as previously described (28). Briefly, the gene of CBD together with the linker domain was synthesized and then inserted into pET-32a (Novage, USA). The vector was named pET-32a-CBD. A human PTHrP DNA encoding a mature form was inserted into the pET-32a and pET-32a-CBD vector, and the recombinant expression plasimd was named pET-CBD-PTHrP (with CBD) and pET-NAT-PTHrP (without CBD). They both contained a $6 \mathrm{X}$ His purification tag for purification and detection in the subsequent experiments. Both of the plasmids were transformed into Escherichia coli Rosetta (DE3) for expressing the protein. E. coli was induced with $0.2 \mathrm{mM}$ isopropyl b-D-thiogalactopyranoside (IPTG) at $25^{\circ} \mathrm{C}$ for $1 \mathrm{~h}$. The recombinant fusion proteins were isolated as soluble bodies. The purity and yields of recombinant proteins were analyzed by $10 \%$ SDS-polyacrylamide gel electrophoresis (SDS-PAGE).

Collagen binding assay. The comparison of binding ability of these 2 factors to collagen was studied using modified solid phase binding assays as previously described (29). Collagen member (0.1 mg) (Zenghai Bio, Shandong, China) was neutralized and added to 96 -well plates $(1 \mathrm{mg} / \mathrm{well})$, and washed 3 times. Wells were blocked with $200 \mu$ l bovine serum albumin (BSA) $(2.5 \mathrm{mg} / \mathrm{ml})$ in phosphate-buffered saline (PBS) plus $0.1 \%$ Tween-20 for $1 \mathrm{~h}$ at room temperature (RT). After washing the wells once with PBS, 50- $\mu 1$ aliquots of the recombinant proteins diluted in PBS were added for $1 \mathrm{~h}$ at $37^{\circ} \mathrm{C}$ with a series of concentrations of $0.156-10 \mathrm{mM}$. Wells were washed 2 times with PBS, and 50- $\mu 1$ aliquots of mouse anti-polyhistidine monoclonal antibody (1:1,000 dilution) were added for $1 \mathrm{~h}$ at RT. After 3 washes as above, $100-\mu 1$ aliquots of sheep anti-mouse-alkaline phosphatase antibody (1:10,000 dilutions) were added for $1 \mathrm{~h}$ at RT, followed by 3 washes as above. Bound proteins were detected with $100 \mu \mathrm{l} /$ well $2 \mathrm{mg} / \mathrm{ml}$ p-nitrophenyl phosphate hexahydrate (p-NPP; Ameresco) in alkaline phosphatase buffer $(100 \mathrm{mM}$ Tris-HC, $100 \mathrm{mM} \mathrm{NaCl}, 10 \mathrm{mM} \mathrm{MgCl}$, $\mathrm{pH}$ 9.6) for $10 \mathrm{~min}$. The reactions were stopped with $0.2 \mathrm{M} \mathrm{NaOH}(100 \mu \mathrm{l} /$ well). One hundred-microliter solutions were then transported to a new 96-well plate. The plate was read in an ELISA reader at a wavelength of $405 \mathrm{~nm}$. All binding assays were carried out in duplicate, and values showed $<15 \%$ difference for the same plate.

Induction of in vitro chondrogenesis. To induce chondrogenesis, in vitro pellet cultures were carried out using $2.5 \times 10^{5}$ BM-MSCs at passage 3 in chondrogenic differentiation medium (Cyagen, USA). From the 14th day of culture, subsets of pellets were additionally treated with PTHrP $(100 \mathrm{ng} / \mathrm{ml})$ and CBD-PTHrP (100 ng/ml), and following 2 additional weeks of in vitro culture in their respective media, the pellets were harvested for analysis. For pellet cultures, $0.5 \mathrm{ml}$ of the cell suspension was aliquoted into $15-\mathrm{ml}$ polypropylene centrifuge tubes, and spun in a bench top centrifuge at $150 \mathrm{xg}$ for $5 \mathrm{~min}$. Tubes were incubated in $5 \% \mathrm{CO}_{2}$ atmosphere for up to 4 weeks. Caps of tubes were loosened in order to allow air exchange. The medium was changed every 2 days.

RNA isolated and real-time PCR analysis. RNA was isolated using the standard guanidine isothiocyanate TRIzol reagent (Invitrogen). Isolated RNA samples were converted to cDNA using Rotor-Gene SYBR Green RT-PCR kit (Qiagen) and oligo(dT) primers. All PCR reactions were performed using ABI system in standard $25-\mu 1$ reaction volumes containing $5 \mu \mathrm{l}$ RNA, $0.5 \mu \mathrm{l}$ of $100 \mathrm{mM}$ sense and $0.5 \mu \mathrm{l}$ of $100 \mathrm{mM}$ antisense primer, 12.5 $\mu$ 1 Rotor-Gene SYBR-Green PCR Master Mix (Qiagen) and 6.5 $\mu \mathrm{l}$ RNA-free $\mathrm{ddH}_{2} \mathrm{O}$. The expression of the following genes were examined: collagen type I (COL1A1), collagen type II (COL2A1), collagen type X (COL10A1), Sox-9 and glyceraldehyde-3-phosphate dehydrogenase (GAPDH) used as a housekeeping gene. The primers used for amplification were: collagen type I, 5'-CCG CCGCTTCACCTACAGC-3' and 5'-TTTTGTATTCAA TCACTGTCTTGCC-3'; collagen type II, 5'-CCGAATAGC AGGTTCACGTACA-3' and 5'-CGATAACAGTCTTGC CCCACTT-3'; collagen type X, 5'-AAAGGCCCACTACCC AACAC-3' and 5'-CTTCCGTAGCCTGGTTTTCC-3'; Sox-9, 5'-CACACAGCTCACTCGACCTTG-3' and 5'-TTCGGT TATTTTTAGGATCATCTCG-3'. 

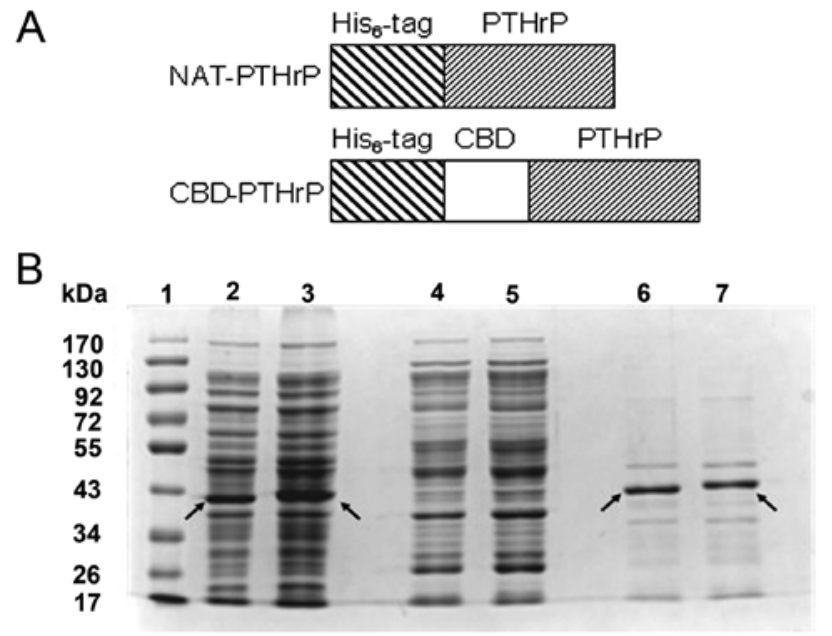

Figure 1. Expression and purification of NAT-PTHrP and CBD-PTHrP. (A) Scheme of protein expression constructs. (B) Expression of NAT-PTHrP and CBD-PTHrP soluble proteins as detected by SDS-PAGE. Lane 1, molecular weight markers; lanes 2, 4 and 6, total soluble proteins of $E$. coli DE3/ pET-NAT-PTHrP after induction, penetration fluid and purified protein of NAT-PTHrP, respectively. Lanes 3, 5 and 7, total soluble proteins of $E$. coli DE3/ pET-CBD-PTHrP after induction, penetration fluid and purified protein of CBD-PTHrP, respectively. The arrows indicate the bands of the target proteins.

Collagen extraction and western blotting. Three pellets were homogenized and subjected to pepsin digestion overnight at $4^{\circ} \mathrm{C}(0.5 \mathrm{M}$ acetic acid, $0.2 \mathrm{M} \mathrm{NaCl}$ and $2.5 \mathrm{mg} / \mathrm{ml}$ of pepsin). The $\mathrm{pH}$ was then adjusted to a neutral $\mathrm{pH} 7.0$ with $1 \mathrm{M}$ Tris Base prior to extraction of the collagens with $4.5 \mathrm{M} \mathrm{NaCl}$ (overnight at $4^{\circ} \mathrm{C}$ ). The following day, the extracted collagens were pelleted by centrifugation at $16,000 \mathrm{xg}$ at $4^{\circ} \mathrm{C}$ for $30 \mathrm{~min}$ and subsequently precipitated with $400 \mu \mathrm{l}$ of precipitation buffer $(0.4 \mathrm{M} \mathrm{NaCl}$ and $0.1 \mathrm{M}$ Tris Base, $\mathrm{pH} 7.4)$ and $1,200 \mathrm{~g}$ of ethanol/sample for $4 \mathrm{~h}$ at $-20^{\circ} \mathrm{C}$. The precipitated collagens were pelleted by centrifugation at $16,000 \mathrm{x}$ g for $30 \mathrm{~min}$ at $4^{\circ} \mathrm{C}$ and resolved in $50 \mu \mathrm{l}$ of RIPA lysis buffer (1\% Triton X-100, $150 \mathrm{mM} \mathrm{NaCl}$ and $50 \mathrm{mM}$ Tris, $\mathrm{pH} 8.0)$. The proteins $(20 \mu \mathrm{g})$ were separated by SDS-PAGE and electronically transferred onto a polyvinylidene difluoride membrane (Millipore, Bedford, MA, USA). After blocking, the membranes were incubated with the recommended dilution primary antibodies against collagen I, collagen II, collagen X, Sox-9 (Millipore) and GAPDH (Santa Cruz Biotechnology, Inc., Santa Cruz, CA, USA), followed by incubation with peroxidase-conjugated secondary antibodies (Abcam, Cambridge, MA, USA). Peroxidase-labeled bands were visualized using an ECL kit (Millipore).

Histological analysis. After 4 weeks of culture, pellets were fixed in $4 \%$ paraformaldehyde solution for $4 \mathrm{~h}$, dehydrated with $100 \%$ ethanol, washed with xylene, and embedded in paraffin. Sections $(4 \mu \mathrm{m})$ were cut from paraffin blocks and coated on APES-treated glass slides. Safranin-O staining for proteoglycan and immunohistochemistry for collagen types I, II, X and Sox-9 (Millipore) were then performed. For Safranin-O staining, sections were deparaffinized with xylene and ethanol, aqueous Safranin-O (0.1\%) (Sigma, USA) was applied for $20 \mathrm{~min}$, and then sections were washed with distilled water. For immunohistochemistry, sections were deparaffinized in xylene, treated with a graded series of alcohol [100, 95 and 80\% ethanol/double-distilled $\left.\mathrm{H}_{2} \mathrm{O}(\mathrm{v} / \mathrm{v})\right]$, and rehydrated in PBS (pH 7.4). Endogenous peroxide was blocked with $3 \% \mathrm{H}_{2} \mathrm{O}_{2}$ for 10 min. After PBS washes, slides were blocked with 5\% normal goat serum in PBS for $15 \mathrm{~min}$ at RT followed by incubation with primary anti-collagen I (1:100), anti-collagen II (1:500), anti-collagen X (1:400) or anti-Sox-9 (1:400) antibody in blocking solution overnight at $4^{\circ} \mathrm{C}$. All slides were subsequently incubated with a 1:200 dilution of biotin-conjugated goat anti-mouse, or goat anti-rabbit secondary antibody for $15 \mathrm{~min}$ at $37^{\circ} \mathrm{C}$ and the streptavidinbiotin complex at $37^{\circ} \mathrm{C}$ for $15 \mathrm{~min}$. The immunoreaction was visualized using diaminobenzidine (DAB) peroxide solution, and cellular nuclei were counterstained with hematoxylin. All specimens were evaluated using an Olympus BX600 microscope and a Spot Fiex camera. Control samples exposed to the secondary antibody alone showed no specific staining.

Statistical analysis. Data are expressed as the means \pm SD. Statistical analysis was performed using the Student's test for comparing 2 groups and by ANOVA for multiple group comparisons. $\mathrm{P}<0.05$ was taken to indicate a statistically significant result. The Statistics Analysis System was used for all statistical analyses.

\section{Results}

CBD-PTHrP expression and purification. Western blotting showed that $E$. coli expressed the recombinant proteins CBD-PTHrP and NAT-PTHrP when induced by IPTG (Fig. 1). The total soluble protein was purified by $6 \mathrm{X}$ His purification tag, and the purified proteins were then diluted in PBS for subsequent experiments.

$C B D-P T H r P$ specifically binds to collagen. The binding abilities of NAT-PTHrP and CBD-PTHrP to collagen were then studied in vitro through collagen-based ELISAs. As shown in Fig. 2A, at each concentration, the OD405 value in the CBD-PTHrP group was higher than that in the NAT-PTHrP group, suggesting that more proteins bound to collagen during the ELISA assay. 

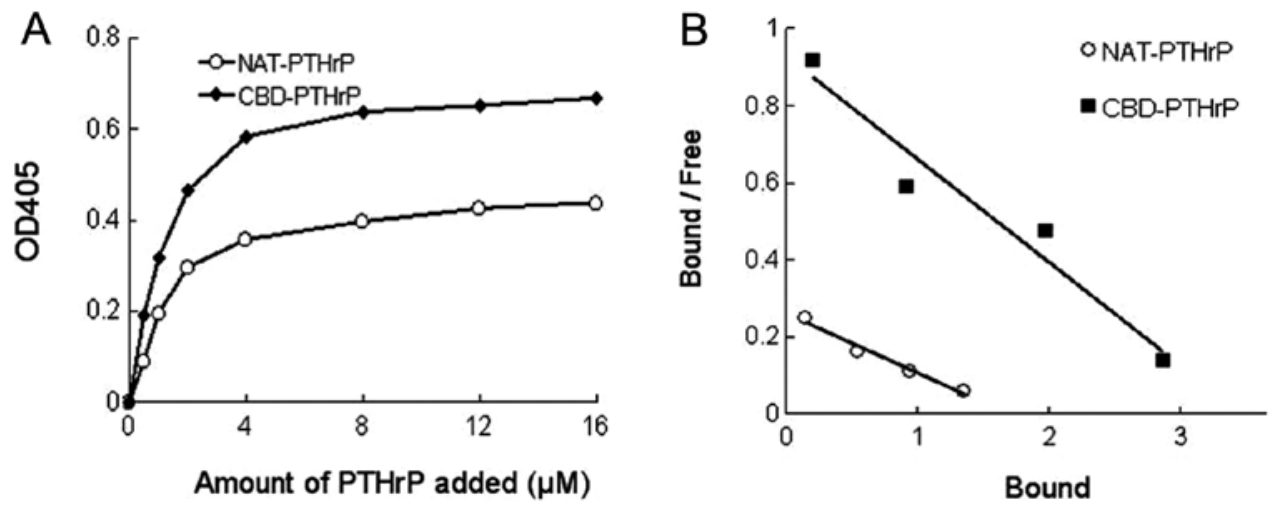

Figure 2. Binding curves of NAT-PTHrP and CBD-PTHrP to native collagen in vitro. (A) Different concentrations of the two types of PTHrP were added to collagen-coated 96 wells. Binding was measured by the color development at $405 \mathrm{~nm}$ after ELISA. (B) Data derived using Scatchard analysis to determine Kd values for collagen interaction with NAT-PTHrP and CBD-PTHrP. Slope of each line $=-1 / \mathrm{Kd}$.
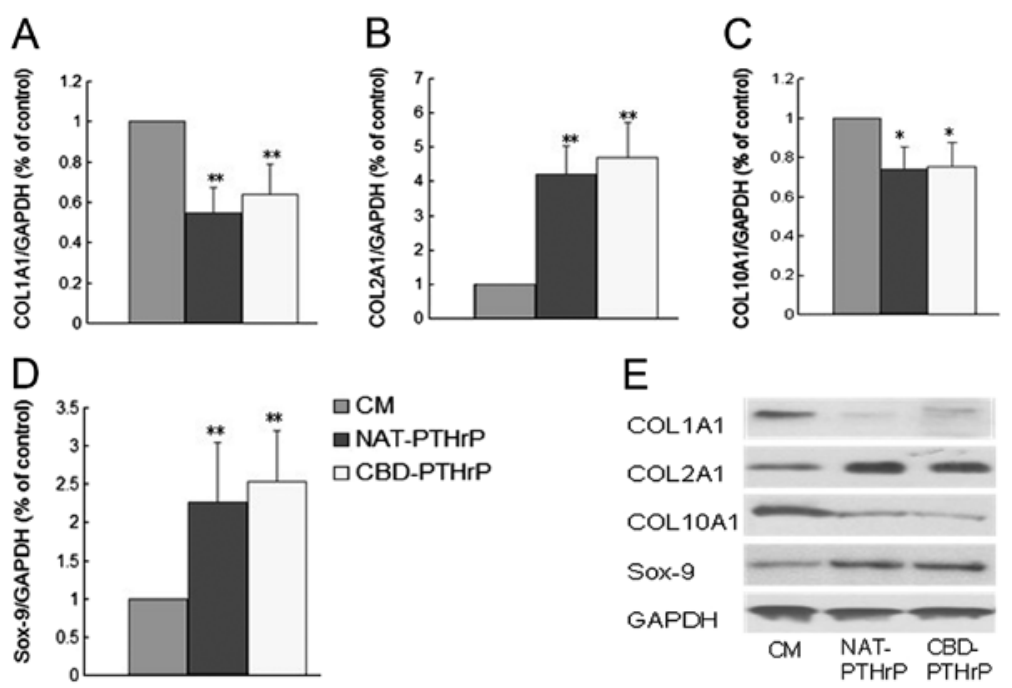

Figure 3. Reverse transcription and real-time PCR of the cells after 4 weeks of culture. The mRNA expression of (A) COL1A1, (B) COL2A1, (C) COL10A1 and (D) Sox-9 were measured and normalized vs. GAPDH. Values are expressed as fold change relative to the control. (E) The protein expression of COL1A1, COL2A1, COL10A1 and Sox-9 was measured by western blotting. Equal amount of protein was loaded on each gel lane. (CM, BM-MSCs treated with their respective chondrogenic medium). The bars represent means \pm SEM $\left(n=3:{ }^{*} \mathrm{P}<0.05,{ }^{* *} \mathrm{P}<0.01\right)$.

Based on the binding curve, the dissociation constant $\mathrm{Kd}$ values for the 2 types of PTHrP binding to collagen $(1 \mathrm{mg}$ ) were calculated by Scatchard analysis (Fig 2B). At each concentration, the ratio of bound counts to unbound counts was plotted against the amount of bound protein. The slope of the resulting straight line was $-1 / \mathrm{Kd}$. The $\mathrm{Kd}$ value for the binding of NAT-PTHrP and CBD-PTHrP to $1 \mathrm{mg}$ collagen was 0.725 and $0.291 \mu \mathrm{M}$, respectively. The lower $\mathrm{Kd}$ value indicated that the protein had a higher binding ability to collagen. Thus, the results clearly demonstrated that CBD-PTHrP possessed stronger collagen-binding capacity vs. NAT-NGF.

Expression of COL1A1, COL2A1, COL10A1 and Sox-9 as determined by $q R T-P C R$ and western blotting. In BM-MSCs, the expression levels of COL1A1, COL2A1, COL10A1 and Sox-9 were determined by qRT-PCR and western blotting at the mRNA and protein levels, respectively. The expression of COL1A1 decreased by $45.38 \%(\mathrm{P}<0.01)$ and $40.81 \%$ $(\mathrm{P}<0.01)$ after treatment with $100 \mathrm{ng} / \mathrm{ml}$ NAT-PTHrP and
CBD-PTHrP, respectively (Fig. 3A). Meantime, Sox-9 mRNA (Fig. 3D), the master gene of chondrogenesis, increased from 1 to $2.3-(\mathrm{P}<0.01)$ and 2.5 -fold $(\mathrm{P}<0.01)$ when compared to the untreated control following treatment with $100 \mathrm{ng} / \mathrm{ml}$ NAT-PTHrP and CBD-PTHrP. The expression of COL2A1 dramatically increased to 4.2 -fold $(\mathrm{P}<0.01)$ following $100 \mathrm{ng} /$ $\mathrm{ml}$ of NAT-PTHrP and 4.7-fold $(\mathrm{P}<0.01)$ following $100 \mathrm{ng} /$ $\mathrm{ml}$ of CBD-PTHrP (Fig. 2B). COL10A1, the marker of hypertrophic chondrocytes, decreased $25.86 \%(\mathrm{P}<0.05)$ following $100 \mathrm{ng} / \mathrm{ml}$ of NAT-PTHrP and $24.79 \%(\mathrm{P}<0.05)$ following $100 \mathrm{ng} / \mathrm{ml}$ of CBD-PTHrP (Fig. 3C). Western blotting provided similar results as the qRT-PCR. As shown in Fig. 3E, the protein levels of COL2A1 and Sox-9 were significantly increased after treatment with $100 \mathrm{ng} / \mathrm{ml}$ NAT-PTHrP and CBD-PTHrP compared with the untreated group. Meanwhile, the protein expression of COL1A1 and COL10A1 was dramatically inhibited by NAT-PTHrP and CBD-PTHrP. There were no significant differences between NAT-PTHrP and CBD-PTHrP at either the mRNA or the protein level. 


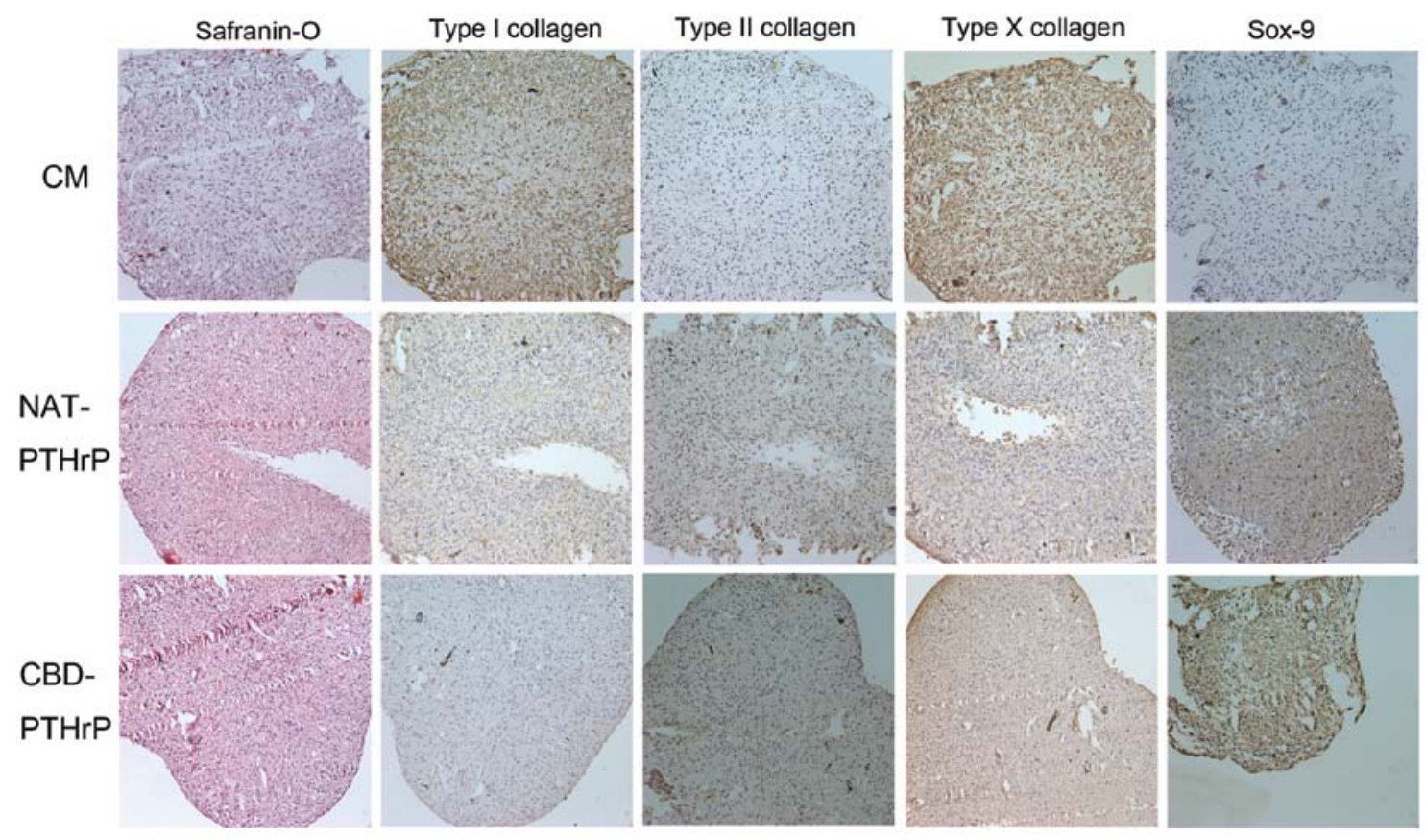

Figure 4. Histological findings of the pellets after 4 weeks of culture. Safranin-O staining (x200), immunohistochemistry (x200) for type I, II and X collagen and Sox-9 of cells cultured in pellets of BM-MSCs. CM, MSCs treated with their respective chondrogenic medium.

Histological findings. Histological findings of Safranin-O and immunohistochemistry for type I, II and X collagens and Sox-9 generally mirrored changes detected by qRT-PCR and western blotting with minor variations. Safranin-O staining showed an increase in the metachromatic staining after NAT-PTHrP and CBD-PTHrP treatment. Type II collagen expression markedly increased in both NAT-PTHrP and CBD-PTHrP treatment groups while type I collagen staining decreased in both treatment groups. Type $\mathrm{X}$ collagen expression decreased after NAT-PTHrP and CBD-PTHrP treatment. Sox-9 protein expression increased dramatically after NAT-PTHrP and CBD-PTHrP treatment in BM-MSCs (Fig. 4).

\section{Discussion}

MSCs are an attractive option for cartilage regeneration because of their abilities to proliferate and their easy accessibility (8-10). However, chondrocyte proliferation and chondrocyte hypertrophy are the main challenges for cartilage regeneration from MSCs $(13,14)$. Previous studies suggest that PTHrP may circumvent these problems by promoting chondrogenesis and suppressing hypertrophy in the growth plate through the PTHrP-Indian hedgehog (IHH) axis (17-20). During chondrocyte induction from MSCs, the cells secrete PTHrP during the early phase of differentiation, until days 14-21, when the mRNA levels for PTHrP declined, whereas those for IHH were upregulated for the remaining weeks of culture (30). PTHrP has been shown to severely reduce type $\mathrm{X}$ collagen expression, AP activity, and cell enlargement of lower sternal chondrocytes from immature chicken, and these molecules are soluble factors produced by articular chondrocytes (31). These results make PTHrP an attractive candidate for chondrocyte induction.
Previous studies have used the PTHrP protein for the purpose of cartilage chondrocyte induction (15). However, simple absorption of PTHrP to the collagen scaffold would allow the diffusion of PTHrP into extracellular fluids, and would rapidly lose its activity. In addition, overexpression of PTHrP by a gene transfer method was also used and induced an arthritic phenotype in articular chondrocytes (32). In the present study, we first engineered PTHrP to construct a collagen-targeting system. Safranin-O staining showed that the recombined protein CBD-PTHrP increased metachromatic staining after treatment (Fig. 4A). At the same time, CBD-PTHrP treatment increased the expression of COL2A1 and Sox-9 and decreased the expression of COL1A1 and COL10A1 at the mRNA and protein levels (Figs. 3 and 4). These results were slightly different than those of Kafienah et al (33) and Kim et al (15). Kafienah et al (33) found that type II collagen expression was unchanged and expression of type I and X collagen was suppressed while Kim et al (15) found that type X collagen expression was gradually decreased, type I collagen expression was suppressed and type II collagen expression was increased after treating chondrogenic cultures of human MSCs with PTHrP.

Tissue engineering of skin, bone, vascular and nerve offers a promising means of producing 3-dimensional neocartilages for clinical treatment (34), and various factors for tissue engineering have been developed, such as bFGF, NGF and PDGF (35-37). However, in clinical practice, factors simply delivered in solution are difficult to be retained at the injured site due to their rapid diffusion in extracellular fluids. Many attempts have been made to overcome the difficulties in using factors as a therapeutic agent. In order to maintain adequate factor concentration, multiple injections are needed. However, this would increase the cost and surgical risks, and may even have adverse effects. Immobilization of bFGF 
on heparin-Sepharose beads prolonged the storage and release (38). Recently, the most abundant component of extracellular matrices, collagen, has been widely used in drug delivery $(39,24)$. Meanwhile, in tissue engineering, many types of collagen-based scaffolds have been fabricated, and they have shown good characteristics in wound repair $(40,41)$. During the process, collagen was found to play an important role in providing a cell anchorage site, mechanical stability and structural guidance. They also provided the interface to respond to physiological and biological changes, and to remodel the extracellular matrix to integrate with the surrounding native tissue $(42,43)$. Moreover, collagen is commonly used as an attractive targeted site for exogenous peptide growth factors. Targeted growth factors on collagen may not only retain its activity and control its diffusion but may also repair injured tissues locally. Nerve growth factor- $\beta$, platelet-derived growth factor and basic fibroblast growth factor have been added with a collagen-binding peptide, and the results have shown that CBD specifically binds to native collagen, and the modified growth factors achieve better repair compared to the native growth factors at the same concentration $(28,44,45)$. In the present study, CBD which is a peptide of 7 amino acids was first used to engineer PTHrP to specially target the PTHrP to collagen. Our results showed that CBD-PTHrP has a higher binding ability to collagen than NAT-PTHrP (Fig. 2). The ability of CBD-PTHrP to induce chondrogenesis in MSCs was also measured by an in vitro pellet assay. As shown in Figs. 3 and 4, CBD-PTHrP induced chondrogenesis and inhibited chondrocyte differentiation toward hypertrophy as well as NAT-PTHrP. In future studies, the collagen-based scaffolds of bone will be used to determine the ability of CBD-PTHrP to produce MSC-derived tissue-engineered cartilage in vivo.

In conclusion, our study suggests that CBD-PTHrP is an attractive recombined protein for use in cartilage tissue engineering from MSCs. It demonstrated that CBD-PTHrP has a higher binding ability to collagen than NAT-PTHrP and significantly enhances chondrogenesis and suppresses hypertrophy in BM-MSCs. Further investigations are warranted to confirm the ability of CBD-PTHrP to produce MSC-derived tissue-engineered cartilage in vivo based on collagen-based scaffolds of bone.

\section{Acknowledgements}

This study was supported by the National Natural Science Foundation of China (nos. 21002018 and 81071498).

\section{References}

1. Cremer MA, Rosloniec EF and Kang AH: The cartilage collagens: a review of heir structure, organization, and role in the pathogenesis of experimental arthritis in animals and in human rheumatic disease. J Mol Med 76: 275-288, 1998.

2. Madry H, van Dijk CN and Mueller-Gerbl M: The basic science of the subchondral bone. Knee Surg Sports Traumatol Arthrosc 18: 419-433, 2010.

3. Jackson DW, Simon TM and Aberman HM: Symptomatic articular cartilage degeneration: the impact in the new millennium. Clin Orthop Relat Res (Suppl): S14-S25, 2001

4. Gomoll AH, Farr J, Gillogly SD, Kercher JS and Minas T: Surgical management of articular cartilage defects of the knee. Instr Course Lect 60: 461-483, 2011.
5. Agnesi F, Amrami KK, Frigo CA and Kaufman KR: Comparison of cartilage thickness with radiologic grade of knee osteoarthritis. Skeletal Radiol 37: 639-643, 2008.

6. Bae WC, Temple MM, Amiel D, Coutts RD, Niederauer GG and Sah RL: Indentation testing of human cartilage: sensitivity to articular surface degeneration. Arthritis Rheum 48: 3382-3394, 2003.

7. Magnussen RA, Dunn WR, Carey JL and Spindler KP: Treatment of focal articular cartilage defects in the knee: a systematic review. Clin Orthop Relat Res 466: 952-962, 2008.

8. Cucchiarini M and Madry H: Gene therapy for cartilage defects. J Gene Med 7: 1495-1509, 2005.

9. Charbord P: Bone marrow mesenchymal stem cells: historical overview and concepts. Hum Gene Ther 21: 1045-1056, 2010.

10. Pittenger MF, Mackay AM, Beck SC, et al: Multilineage potential of adult human mesenchymal stem cells. Science 284 : 143-147, 1999.

11. Grogan SP, Miyaki S, Asahara H, D'Lima DD and Lotz MK: Mesenchymal progenitor cell markers in human articular cartilage: normal distribution and changes in osteoarthritis. Arthritis Res Ther 11: R85, 2009.

12. Koelling S, Kruegel J, Irmer M, Path JR, Sadowski B, Miro X and Miosge N: Migratory chondrogenic progenitor cells from repair tissue during the later stages of human osteoarthritis. Cell Stem Cell 4: 324-335, 2009.

13. Barry F, Boynton RE, Liu B and Murphy JM: Chondrogenic differentiation of mesenchymal stem cells from bone marrow; differentiation-dependent gene expression of matrix components. Exp Cell Res 268: 189-200, 2001.

14. Pelttari K, Winter A, Steck E, et al: Premature induction of hypertrophy during in vitro chondrogenesis of human mesenchymal stem cells correlates with calcification and vascular invasion after ectopic transplantation in SCID mice. Arthritis Rheum 54 : 3254-3266, 2006.

15. Kim YJ, Kim HJ and Im GI: PTHrP promotes chondrogenesis and suppresses hypertrophy from both bone marrow-derived and adipose tissue-derived MSCs. Biochem Biophys Res Commun 373: 104-108, 2008.

16. Suva LJ, Winslow GA, Wettenhall RE, et al: A parathyroid hormone-related protein implicated in malignant hypercalcemia: cloning and expression. Science 237: 893-896, 1987.

17. Kronenberg HM: PTHrP and skeletal development. Ann NY Acad Sci 1068: 1-13, 2006.

18. Kobayashi T, Soegiarto DW, Yang Y, et al: Indian hedgehog stimulates periarticular chondrocyte differentiation to regulate growth plate length independently of PTHrP. J Clin Invest 115: 1734-1742, 2005 .

19. Huang W, Chung UI, Kronenberg HM and de Crombrugghe B: The chondrogenic transcription factor Sox 9 is a target of signaling by the parathyroid hormone-related peptide in the growth plate of endochondral bones. Proc Natl Acad Sci USA 98: 160-165, 2001.

20. Amling M, Neff L, Tanaka S, et al: Bcl-2 lies downstream of parathyroid hormone-related peptide in a signaling pathway that regulates chondrocyte maturation during skeletal development. J Cell Biol 136: 205-213, 1997.

21. Yurchenco PD, Smirnov S and Mathus T: Analysis of basement membrane self-assembly and cellular interactions with native and recombinant glycoproteins. Methods Cell Biol 69: 111-144, 2002.

22. Eyre DR: Collagen of articular cartilage. Clin Orthop Relat Res (427 Suppl): S118-S122, 2004.

23. Andrades JA, Han B, Becerra J, Sorgente N, Hall FL and Nimni ME: A recombinant human TGF-betal fusion protein with collagen-binding domain promotes migration, growth, and differentiation of bone marrow mesenchymal cells. Exp Cell Res 250: 485-498, 1999.

24. Nishi N, Matsushita O, Yuube K, Miyanaka H, Okabe A and Wada F: Collagen-binding growth factors: production and characterization of functional fusion proteins having a collagen-binding domain. Proc Natl Acad Sci USA 95: 7018-7023, 1998

25. Ishikawa T, Terai $\mathrm{H}$, Yamamoto $\mathrm{T}$, Harada $\mathrm{K}$ and Kitajima $\mathrm{T}$ : Delivery of a growth factor fusion protein having collagenbinding activity to wound tissues. Artif Organs 27: 147-154, 2003.

26. Im GI, Shin YW and Lee KB: Do adipose tissue-derived mesenchymal stem cells have the same osteogenic and chondrogenic potential as bone marrow-derived cells? Osteoarthritis Cartilage 13: 845-853, 2005 . 
27. Im GI, Jung NH and Tae SK: Chondrogenic differentiation of mesenchymal stem cells isolated from patients in late adulthood: the optimal conditions of growth factors. Tissue Eng 12: 527-536, 2006

28. Sun WJ, Sun CK, Lin H, et al: The effect of collagen-binding NGF-beta on the promotion of sciatic nerve regeneration in a rat sciatic nerve crush injury model. Biomaterials 30: 4649-4656, 2009.

29. SunW, Lin H, Chen B, ZhaoW, Zhao Y and Dai J: Promotion of peripheral nerve growth by collagen scaffolds loaded with collagen-targeting human nerve growth factor-beta. J Biomed Mater Res A 83: 1054-1061, 2007.

30. Fischer J, Dickhut A, Rickert M and Richter W: Human articular chondrocytes secrete parathyroid hormone-related protein and inhibit hypertrophy of mesenchymal stem cells in coculture during chondrogenesis. Arthritis Rheum 62: 2696-2706, 2010.

31. Schmid TM and Linsenmayer TF: Immunohistochemical localization of short chain cartilage collagen (type X) in avian tissues. J Cell Biol 100: 598-605, 1985.

32. Wang D, Taboas JM and Tuan RS: PTHrP overexpression partially inhibits a mechanical strain-induced arthritic phenotype in chondrocytes. Osteoarthritis Cartilage 19: 213-221, 2011.

33. Kafienah W, Mistry S, Dickinson SC, Sims TJ, Learmonth I and Hollander AP: Three-dimensional cartilage tissue engineering using adult stem cells from osteoarthritis patients. Arthritis Rheum 56: 177-187, 2007.

34. Caplan AI: Mesenchymal stem cells: cell-based reconstructive therapy in orthopedics. Tissue Eng 11: 1198-1211, 2005.

35. Heldin CH and Westermark B: Mechanism of action and in vivo role of platelet-derived growth factor. Physiol Rev 79: 1283-1316, 1999.
36. Otto D, Unsicker K and Grothe C: Pharmacological effects of nerve growth factor and fibroblast growth factor applied to the transectioned sciatic nerve on neuron death in adult rat dorsal root ganglia. Neurosci Lett 83: 156-160, 1987.

37. Ishihara M, Obara K, Ishizuka T, et al: Controlled release of fibroblast growth factors and heparin from photocrosslinked chitosan hydrogels and subsequent effect on in vivo vascularization. J Biomed Mater Res A 64: 551-559, 2003.

38. Cai S, Liu Y, Zheng Shu X and Prestwich GD: Injectable glycosaminoglycan hydrogels for controlled release of human basic fibroblast growth factor. Biomaterials 26: 6054-6067, 2005.

39. Andrades JA, Wu LT, Hall FL, Nimni ME and Becerra J: Engineering, expression, and renaturation of a collagen-targeted human bFGF fusion protein. Growth Factors 18: 261-275, 2001.

40. Li X, Feng Q, Liu X, Dong W and Cui F: Collagen-based implants reinforced by chitin fibres in a goat shank bone defect model. Biomaterials 27: 1917-1923, 2006.

41. Park SN, Kim JK and Suh H: Evaluation of antibiotic-loaded collagen-hyaluronic acid matrix as a skin substitute. Biomaterials 25: 3689-3698, 2004.

42. Rose FR and Oreffo RO: Bone tissue engineering: hope vs. hype. Biochem Biophys Res Commun 292: 1-7, 2002.

43. Chapekar MS: Tissue engineering: challenges and opportunities. J Biomed Mater Res 53: 617-620, 2000.

44. Zhao WX, Chen B, Li X, et al: Vascularization and cellularization of collagen scaffolds incorporated with two different collagen-targeting human basic fibroblast growth factors. J Biomed Mater Res A 82: 630-636, 2007.

45. Lin H, Chen B, Sun W, Zhao W, Zhao Y and Dai Y: The effect of collagen-targeting platelet-derived growth factor on cellularization and vascularization of collagen scaffolds. Biomaterials 27: $5708-5714,2006$ 